\title{
Osteopontin regulates growth and migration of human nasopharyngeal cancer cells
}

\author{
GUANG YANG $^{1,2}$, YAN ZHANG ${ }^{3}$, JING WU $^{1}$, JIANJUN XIONG $^{1}$, HAIXIA DENG $^{4}$, \\ JIALI WANG ${ }^{1}$, CHANGFU YANG $^{1}$ and ZHENYU ZHU ${ }^{1,2}$ \\ ${ }^{1}$ Department of Biochemistry and Molecular Biology, Zhongshan Medical College, Sun Yat-sen University; \\ ${ }^{2}$ DaAn Gene Co., Ltd., Sun Yat-sen University, Guangzhou; ${ }^{3}$ The Center of Prenatal Diagnosis, \\ Maternal and Child Health Hospital of Guangdong Province; ${ }^{4}$ Department of Breast Surgery, \\ Sun Yat-Sen Memorial Hospital, Sun Yat-Sen University, Guangzhou, Guangdong, P.R. China
}

Received March 24, 2011; Accepted July 13, 2011

DOI: $10.3892 / \mathrm{mmr} .2011 .538$

\begin{abstract}
Naspharyngeal carcinoma (NPC) is a common malignant tumor. Radiotherapy is the main treatment for patients with early-stage NPC. Some chemotherapeutic methods have been used to delay the progression of NPC during the later stages. Osteopontin (OPN), a secreted phosphoglycoprotein, has been shown to be involved in cell proliferation, migration and invasion in a variety of tumor models. However, the effect of OPN on the proliferation and migration activity of human NPC cells is still unknown. In this study, we detected the expression of OPN in NPC CNE-2 cells, and investigated the roles of OPN in CNE-2 cells. Our results show that the down-regulation of OPN inhibits the growth and migration of CNE-2 cells. Additionally, our results indicate that the cell number in the G2 phase can be regulated by OPN. We further demonstrate that OPN shRNA down-regulates the expression of MMP-2 and MMP-9, and that OPN overexpression up-regulates the expression of MMP-2 and MMP-9.
\end{abstract}

\section{Introduction}

Human nasopharyngeal carcinoma (NPC) is a fatal disease very common in southern China and Southeast Asia (1). Approximately $80 \%$ of NPC cases in the world occur in China according to the WHO data. Almost $95 \%$ of the histotypes of clinical NPC are poorly differentiated squamous carcinoma and are sensitive to X-ray treatments (2). Radiotherapy is the main treatment for patients with early-stage NPC. However,

Correspondence to: Professor Zhenyu Zhu, Department of Biochemistry and Molecular Biology, Zhongshan Medical College, Sun Yat-sen University, 74 Zhongshan 2 Road, Guangzhou, Guangdong 510080, P.R. China

E-mail: yangpecker2@yahoo.com.cn

Key words: osteopontin, human nasopharyngeal cancer, growth, migration, RNA interference the 5-year survival rate is still low and the incidence of relapses remains high (3). Therefore, identification of the target genes associated with NPC progression is necessary in order to improve the survival of patients with NPC.

Osteopontin (OPN) is a secreted arginine-glycine-asparic acid (RGD)-containing phosphoprotein and it contains a predicted thrombin cleavage site (4). Substantial data have shown that OPN plays important roles in tumorigenesis, invasion and metastases by binding to several integrins and CD44 variants in various tumor models (5-7). Certain reports have also proven that OPN could be involved in cell survival (8-10). Functionally, the down-regulation of OPN decreases the motility and invasiveness of tumor cells. The up-regulation of OPN increases cell invasiveness and plasminogen activator expression in human mammary epithelial cells (11). OPN enhances the migration and invasion of malignant tumor cells possibly by regulating the activities of MMP-2 and MMP-9, which degrade the extracellular matrix $(9,10,12-14)$. It has been reported that the mean plasma OPN level is significantly higher in NPC patients than in normal controls, and that a high pretreatment plasma osteopontin level is a significant predictor of poor response to radiotherapy $(15,16)$. It has also been shown that OPN knockdown can decrease the number of cells in the G2/M phase $(9,10)$. However, the role of OPN in the progression of NPC is still undefined, and therefore, it is imperative to evaluate the roles of OPN in human NPC cells, as well as the related mechanisms.

\section{Materials and methods}

Cell culture. The poorly differentiated human NPC cell line, CNE-2 (stored in our lab), was cultured in high-glucose DMEM (Gibico, BRL, Guangzhou, China) supplemented with $10 \%$ fetal bovine serum at $37^{\circ} \mathrm{C}$ with $5 \% \mathrm{CO}_{2}$.

Construction of vectors. According to a previous report (17), we constructed the RNAi vector, EGFP was amplified from pEGFP-N1, and then subcloned between $K p n \mathrm{I}$ and BamHI of pcDNA3.1(+). The plasmid pLVTHM expressing shRNA (OPN shRNA) against human OPN and the control vector (NC), were a gift from Dr Yang Zhang (Department 


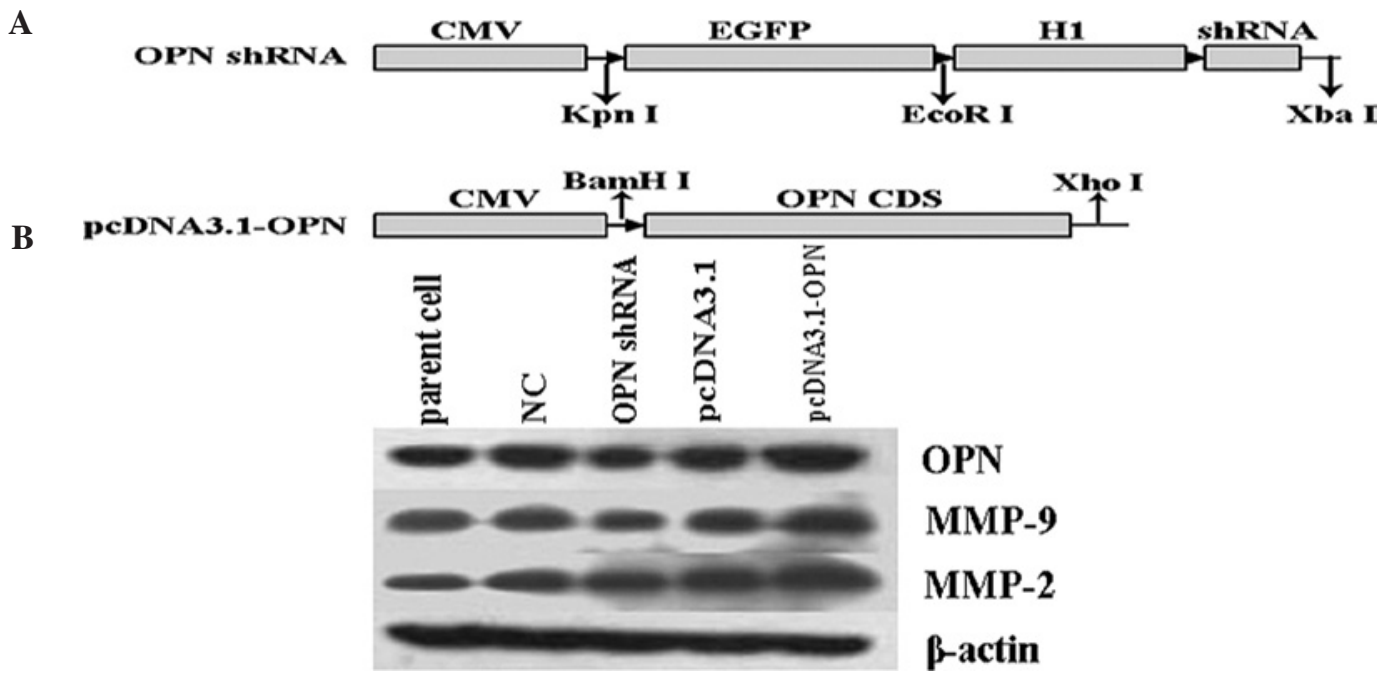

Figure 1. Regulation of OPN, MMP-2 and MMP-9 expressions by the OPN shRNA and the OPN expression vector. (A) Schematic representation of the constructed vectors. CMV, human cytomegalovirus immediate early promoter; EGFP, enhanced green fluorescent protein gene; H1, H1 promoter. (B) Detection of OPN, MMP-2 and MMP-9 in CNE-2 cells. Four CNE-2 cultures transfected independently with NC, OPN shRNA, pcDNA3.1 and pcDNA3.1-OPN were harvested for Western blot analysis to detect the expressions of OPN, MMP-2 and MMP-9. Equivalent loading is controlled by $\beta$-actin detection.

of Biochemistry and Molecular Biology, Zhongshan Medical College, Sun Yat-sen University, China). The sequence targeting OPN mRNA and the control sequence were as follows: OPN shRNA, 5'-CACAGACCCTTCCAAGTAA-3'; and NC, 5'-TAGCGACTAAACACATCAA-3'. The plasmids were digested by EcoRI and $X b a \mathrm{I}$, then the sequence containing the $\mathrm{H} 1$ promoter and dsDNA was ligated between the EcoRI and XbaI sites of the pcDNA3.1(+) vector. To construct the OPN overexpression vector, pcDNA3.1-OPN, the full-length OPN cDNA was subcloned between the BamHI and XhoI sites of pcDNA3.1(+) vector (Fig. 1). Purified plasmids were sequence-verified by Invitrogen (Shanghai, China). The cells were transfected using Lipofectamine reagent (Beyotime, China) according to the manufacturer's instructions.

MTT assay. CNE-2 cells were seeded in 96-well plates at a concentration of 5,000 cells/well in a volume of $150 \mu \mathrm{l}$ of cell culture medium. After $24 \mathrm{~h}$, transfection was performed. The plates were incubated at $37^{\circ} \mathrm{C}$ with $5 \% \mathrm{CO}_{2}$ for $24,48,72$ and $96 \mathrm{~h}$. A sample $(20 \mu \mathrm{l})$ of MTT solution $(5 \mathrm{~g} / \mathrm{l}$, dissolved in PBS) was added to each well and the plates were incubated at $37^{\circ} \mathrm{C}$ for an additional $4 \mathrm{~h}$. The supernatant was discarded, then $150 \mu$ l dimethylsulfoxide were added to dissolve the insoluble MTT formazan. The absorbance values at $570 \mathrm{~nm}$ (A570) were determined on a multi-well plate reader (Tecan).

Migration assay. To determine the cell migration potential, the migration assay was carried out using the Transwell (Costar) system, which allows cells to migrate through an $8-\mu \mathrm{m}$ pore size polycarbonate membrane. Briefly, cells were transfected with constructed vectors for $24 \mathrm{~h}$, and were then trypsinized, washed and resuspended in DMEM with $5 \%$ FCS $\left(5 \times 10^{5}\right.$ cells/ $\mathrm{ml})$. A total amount of $600 \mu \mathrm{l}$ of DMEM containing $10 \%$ FCS was placed into the lower compartment of the well and $100 \mu \mathrm{l}$ of cell suspension in DMEM without FCS were added to the upper compartments. After having being cultured at $37^{\circ} \mathrm{C}$ in a humidified atmosphere of $5 \% \mathrm{CO}_{2}$ for $20 \mathrm{~h}$, membranes were washed with PBS, all cells on the membrane were fixed with cold methanol for $15 \mathrm{~min}$ and stained with crystal violet, then cells remaining on the upper surface of the membrane were removed with a cotton swab. Cells beneath the membrane were counted in 5 high-power microscope fields. Each experiment was performed on 3 Transwells and repeated three times.

Flow cytometry assay. For the analysis of the cell cycle, cells transfected with different vectors for $72 \mathrm{~h}$ were subjected to flow cytometry analysis for chromosomal DNA. DNA labelling was performed using the CycleTEST ${ }^{\text {тм }}$ PLUS DNA reagent kit (BD Biosciences Pharmingen, USA). The samples were then analyzed using a flow cytometer (Beckman Coulter, Brea, CA, USA).

Western blot assay. Cells were transfected for $72 \mathrm{~h}$, then the cells were harvested and lysed for total protein extraction. Protein concentration was determined using the Bio-Rad protein assay kit (Bio-Rad, Shanghai, China). Equal amounts of protein were separated by $12 \%$ SDS-PAGE and transferred onto PVDF membranes. The membranes were rinsed with Tris-buffered saline and Tween (TBST; Probe Co. Ltd., Guangzhou, China) and incubated in blocking buffer (5\% dried milk in PBS) for $1 \mathrm{~h}$ at $37^{\circ} \mathrm{C}$, followed by incubation with primary antibodies at $4^{\circ} \mathrm{C}$ overnight. The polyclonal antibody against OPN (Sigma, USA) was used at a 1,000-fold dilution. Monoclonal antibody against $\beta$-actin (PTG, USA) was used at a 2,000-fold dilution. Antibodies against MMP-2 or MMP-9 (Santa Cruz Biotechnology, Santa Cruz, CA, USA) were used at 300-fold dilution. After being washed with TBST three times, the membranes were incubated with their corresponding secondary antibodies for $1 \mathrm{~h}$. The blots were visualized by the enhanced chemiluminescence detection system (Amersham). The expression of $\beta$-actin was used as the normalization control for protein loading. 


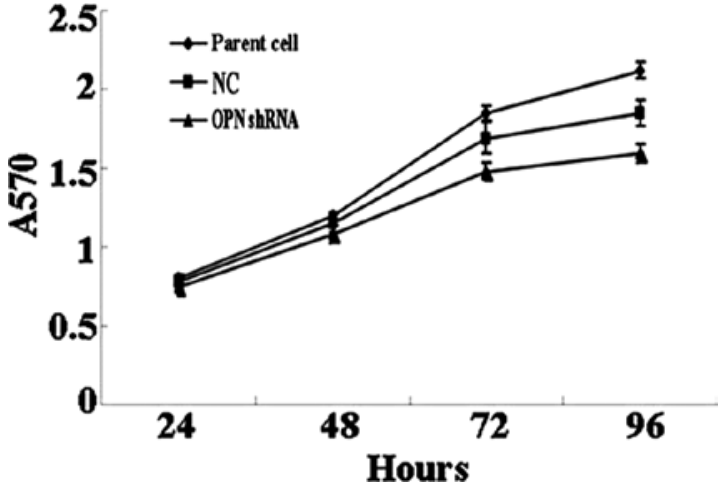

Figure 2. Cell proliferation analysis. CNE-2 cells were seeded in 96-well plates in triplicate for $24 \mathrm{~h}$, and were then transfected with either NC, OPN shRNA, pcDNA3.1 and pcDNA3.1-OPN. MTT assay was performed at the indicated time. Values represent the means \pm SD of 3 wells. " $\mathrm{P}<0.05$, compared with the parent cells.

Statistical analysis. Data were expressed as the means \pm SD. Statistical analyses were performed using the Student's t-test. A value of $\mathrm{P}<0.05$ was considered significant.

\section{Results}

OPN shRNA specifically inhibits OPN expression in CNE-2 cells. Western blot analysis was carried out to inspect OPN protein in CNE-2 cells transfected with the shRNA expression vector or the overexpression vector. As shown in Fig. 1, treatment of CNE-2 cells with OPN shRNA resulted in a significant decrease of OPN expression at the protein level compared with the control vector (NC)-treated CNE-2 cells. The overexpression vector (pcDNA3.1-OPN) transfection markedly increased OPN expression.

Effect of OPN shRNA on CNE-2 cell growth. It has been reported that OPN silencing by RNAi suppresses the prolife- ration of other tumor cells, therefore we examined the effects of OPN on the proliferation of CNE-2 cells. As shown in the Fig. 2 growth curve, OPN shRNA inhibited the growth of CNE-2 cells, especially after 48 h transfection. Interestingly, there was no difference between the cells transfected with the overexpression vector and the control vector.

Regulation of CNE-2 migration ability by OPN expression. We evaluated whether the changed expression of OPN alters the motility of CNE-2 cells using the Transwell system. As shown in Fig. 3, compared to the cells transfected with the related control vectors, we observed that the expression of OPN shRNA had an inhibitory effect on the ability of CNE-2 cells and that transfection with the OPN overexpression vector significantly increased the motility of CNE-2 cells.

Analysis of cell cycle by flow cytometry. Different groups of cells transfected for $72 \mathrm{~h}$ were subjected to flow cytometry analysis. As shown in Fig. 4, the proportion of the G2 phase was decreased in OPN shRNA-transfected cells compared with the control vector-transfected group, and the cell number in the G2 phase showed no change when cells were transfected with pcDNA3.1-OPN. These data suggest that the OPN shRNA suppresses the proliferation of CNE-2 cells by reducing the cell number in the $\mathrm{G} 2$ phase.

Effect of OPN on the expressions of MMP-2 and MMP-9. It has been identified that MMP-2 and MMP-9 are involved in OPN-induced cell proliferation and migration. Therefore, we investigated whether OPN also influences the expressions of MMP-2 and MMP-9 in CNE-2 cells. As shown in Fig. 1, compared with the related control groups, the protein levels of MMP-2 and MMP-9 decreased in the cells transfected with OPN shRNA, but increased in the cells transfected with the OPN overexpression vector. Therefore, these data suggest that MMP-2 and MMP-9 are involved in OPN-induced cell migration in CNE-2 cells.
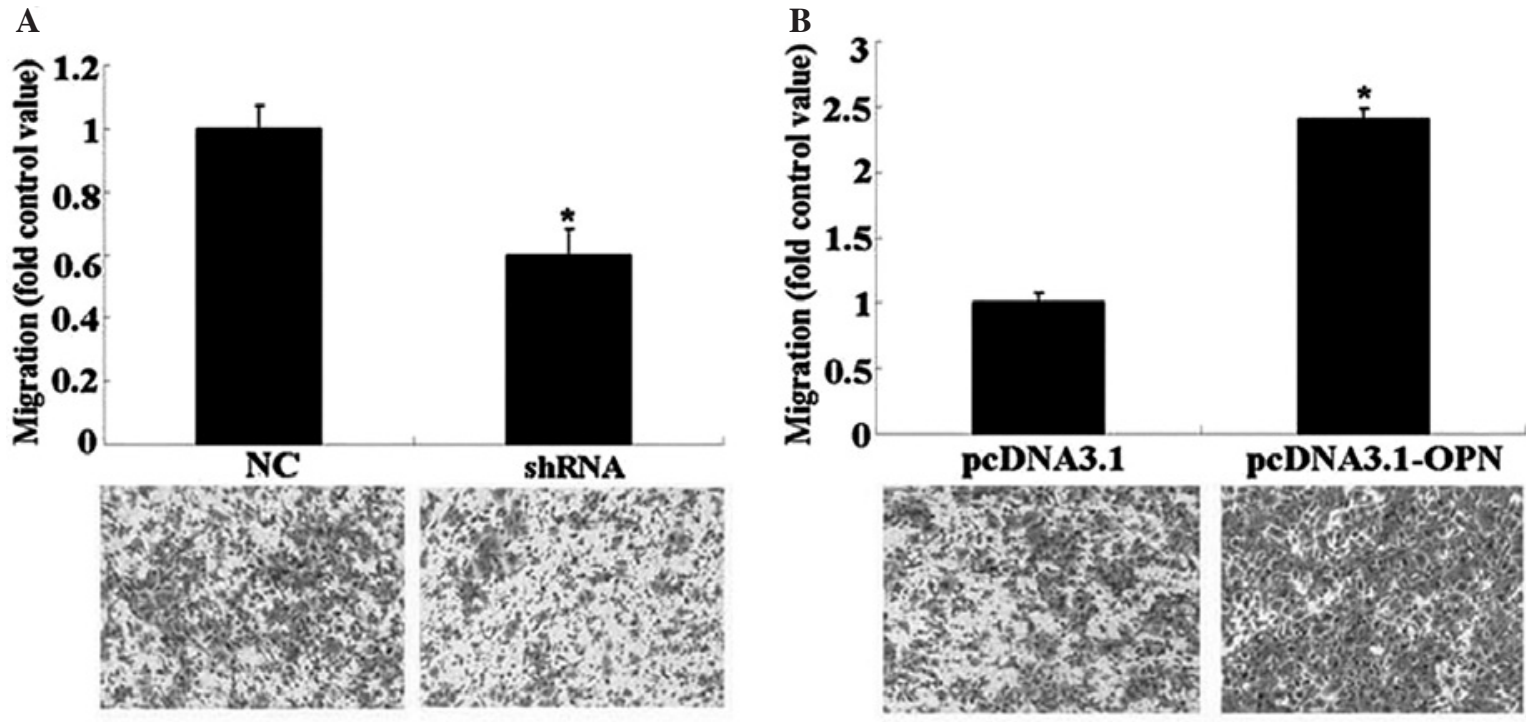

Figure 3. Migration abilities of CNE-2 cells detected by Transwell assay. (A) Cells were transfected with NC or OPN shRNA. (B) Overexpression of OPN in CNE-2 cells significantly promotes the cell migration ability. Data represent the means \pm SD of relative migration vs. the control from 3 independent experiments. ${ }^{~} \mathrm{P}<0.05$, compared with the related control vector-transfected groups. 

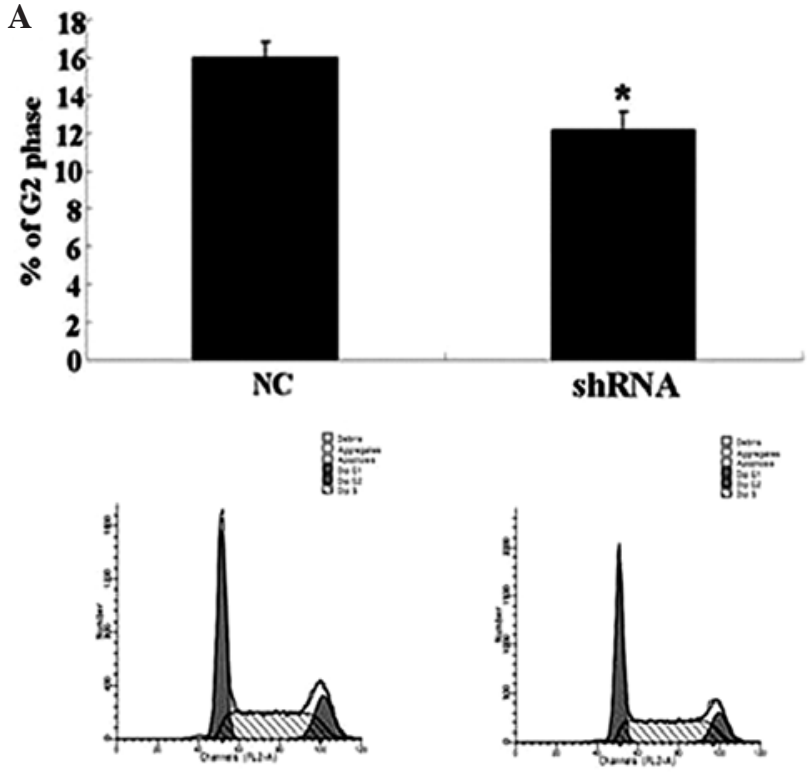

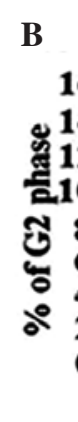

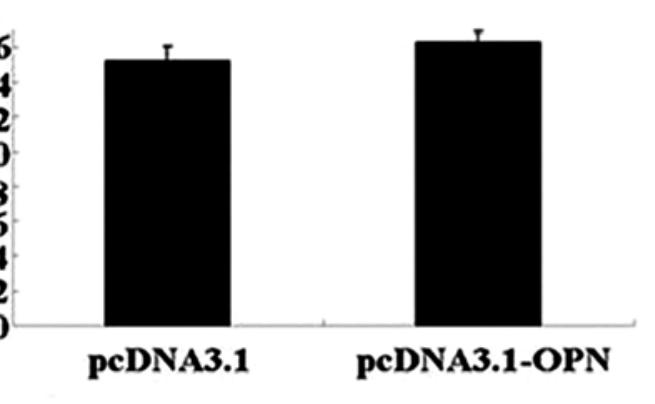

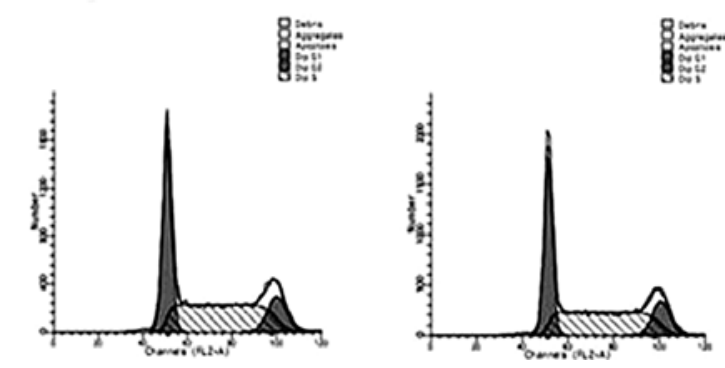

Figure 4. Flow cytometry analysis of the cell cycle. (A and B) The G2 phase of CNE-2 cells transfected with OPN shRNA decreased compared with the control group. However, the cell number in the G2 phase did not increase when cells were transfected with the OPN expression vector. The data represent the means \pm SD of 3 independent experiments. ${ }^{*} \mathrm{P}<0.05$, compared with the control-transfected groups.

\section{Discussion}

Previous studies have demonstrated that OPN plays a crucial role in the growth, invasion and metastasis in a variety of tumors and that many human carcinomas overexpress OPN (18-22). Previous reports have shown that the mean plasma OPN level is significantly higher in NPC patients, and a high OPN level suggests poor response to radiotherapy $(15,16)$. However, the role of OPN in NPC remains poorly understood. In this study, OPN shRNA and the OPN expressing vector were utilized to investigate the influences of OPN on the biological behavior of NPC and related mechanisms. Our data indicate that altering the level of OPN can influence the growth, migration and cell cycle of human nasopharyngeal carcinoma CNE-2 cells. To our knowledge, this is the first report showing a direct link between OPN and NPC cells. RNAi is a highly specific and efficient way of controling the expression of the target gene. However, chemically synthesized siRNA has been shown to have a relatively short half-life to inhibit the target gene $(23,24)$, and thus we used shRNA expression vector-mediated RNAi to achieve a greater inhibitory effect, and the vector expressing OPN was also constructed. The down-regulation of OPN inhibited the growth and migration of CNE-2 cells, and led to a fewer number of cells in the $\mathrm{G} 2$ phase. These data are consistent with recent reports describing the role of OPN in other tumor cells $(9,10)$. The up-regulation of OPN by the OPN expressing vector promotes migration but does not accelerate the proliferation of CNE-2 cells or increase the number of cells in the G2 phase. However, the reason for this, remains unknown. According to the flow cytometry data, there was no difference in the apoptotic ratio between cells transfected with OPN shRNA and those transfected with control shRNA, suggesting that OPN is not involved in the apoptosis of CNE-2 cells.

In this study, we also detected two potential OPN downstream target molecules, MMP-2 and MMP-9, involved in tumor progression and invasion $(14,25)$. Our data on CNE-2 cells indicated that the expressions of MMP-2 and MMP-9 were decreased in the OPN knockdown cells, and significantly increased in the OPN expressing vector-transfected cells. MMP-2 and MMP-9 may play critical roles in the OPN-induced invasion and metastasis of NPC cells. It has been reported that OPN-induced expressions of MMP-2 and MMP-9 are associated with NF- $\mathrm{KB}$ mediated signaling pathways (26). Therefore, the role of NF- $\mathrm{kB}$ and its related protein factors in these processes of NPC cells need to be further elucidated.

In conclusion, our data show that OPN plays a crucial role in the growth and migration of NPC cells. Therefore, gene therapy or small molecule inhibitors targeting OPN may have therapeutic benefit for patients with NPC. Inhibitors targeting OPN may also lead to a decrease in the plasma OPN level and a lower plasma OPN level could make human NPC more sensitive to radiotherapy.

\section{Acknowledgements}

This study was supported by the National Natural Science Foundation of China (Grant no. 30672358 to Z.Z.). We are grateful to Dr Kong at the Department of Inspection of the First Affiliated Hospital of Sun Yat-sen University for his skillful assistance with our flow cytometry analysis.

\section{References}

1. Chang ET and Adami HO: The enigmatic epidemiology of nasopharyngeal carcinoma. Cancer Epidemiol Biomarkers Prev 15: 1765-1777, 2006

2. Guigay J: Advances in nasopharyngeal carcinoma. Curr Opin Oncol 20: 264-269, 2008.

3. Vlantis AC, Tsang RK, Yu BK, Kam MK, Tong MC and Lo PS: Nasopharyngectomy and surgical margin status: a survival analysis. Arch Otolaryngol Head Neck Surg 133: 1296-1301, 2007.

4. Weber GF, Ashkar S, Glimcher MJ and Cantor H: Receptorligand interaction between CD44 and osteopontin (Eta-1). Science 271: 509-512, 1996. 
5. Agrawal D, Chen T, Irby R, et al: Osteopontin identified as lead marker of colon cancer progression using pooled sample expression profiling. J Natl Cancer Inst 94: 513-521, 2002.

6. Rudland PS, Platt-Higgins A, El-Tanani M, et al: Prognostic significance of the metastasis-associated protein osteopontin in human breast cancer. Cancer Res 62: 3417-3427, 2002

7. Coppola D, Szabo M, Boulware D, Muraca P, Alsarraj M, Chambers AF and Yeatman TJ: Correlation of osteopontin protein expression and pathological stage across a wide variety of tumor histologies. Clin Cancer Res 10: 184-190, 2004.

8. Geissinger E, Weisser C, Fischer P, Schartl M and Wellbrock C: Autocrine stimulation by osteopontin contributes to antiapoptotic signalling of melanocytes in dermal collagen. Cancer Res 62: 4820-4828, 2002.

9. Gong M, Lu Z, Fang G, Bi J and Xue X: A small interfering RNA targeting osteopontin as gastric cancer therapeutics. Cancer Letters 272: 148-159, 2008.

10. Liu H, Chen A, Guo F and Yuan L: A short-hairpin RNA targeting osteopontin down-regulates MMP-2 and MMP-9 expressions in prostate cancer PC-3 cells. Cancer Letters 295, 27-37, 2010.

11. Tuck AB, Arsenault DM, O'Malley FP, Hota C, Ling MC Wilson SM and Chambers AF: Osteopontin induces increased invasiveness and plasminogen activator expression of human mammary epithelial cells. Oncogene 18: 4237-4246, 1999.

12. Kim Y and Jeoung D: The cancer/testis antigen CAGE induces MMP-2 through the activation of NF- $\kappa$ B and AP-1. BMB Rep 42: 758-763, 2009

13. Desai B, Ma T, Zhu J and Chellaiah MA: Characterization of the expression of variant and standard CD44 in prostate cancer cells: identification of the possible molecular mechanism of CD44/MMP9 complex formation on the cell surface. J Cell Biochem 108: 272-284, 2009.

14. Castellano G, Malaponte G, Mazzarino MC, et al: Activation of the osteopontin/matrix metalloproteinase-9 pathway correlates with prostate cancer progression. Clin Cancer Res 14: 7470-7480, 2008
15. Wong TS, Kwong DL, Sham J, Wei WI, Kwong YL and Yuen AP: Elevation of plasma osteopontin level in patients with undifferentiated nasopharyngeal carcinoma. Eur J Surg Oncol 31: 555-558, 2005

16. Hui EP, Sung FL, Yu BK, et al: Plasma osteopontin, hypoxia, and response to radiotherapy in nasopharyngeal cancer. Clin Cancer Res 14: 7080-7087, 2008.

17. Cheng J, Huo DH, Kuang DM, Yang J, Zheng L and Zhuang SM: Human macrophages promote the motility and invasiveness of osteopontin-knockdown tumor cells. Cancer Res 67: 5141-5147, 2007.

18. Gotoh M, Sakamoto M, Kanetaka K, Chuuma M and Hirohashi S: Overexpression of osteopontin in hepatocellular carcinoma. Pathol Int 52: 19-24, 2002.

19. Ue T, Yokozaki H, Kitadai Y, Yamamoto S, Yasui W, Ishikawa T and Tahara E: Co-expression of osteopontin and CD44v9 in gastric cancer. Int J Cancer 79: 127-132, 1998.

20. Chambers AF, Wilson SM, Kerkvliet N, O'Malley FP, Harris JF and Casson AG: Osteopontin expression in lung cancer. Lung Cancer 15: 311-323, 1996

21. Pan HW, Ou YH, Peng SY, et al: Overexpression of osteopontin is associated with intrahepatic metastasis, early recurrence, and poorer prognosis of surgically resected hepatocellular carcinoma. Cancer 98: 119-127, 2003.

22. Agrawal D, Chen T, Irby R, et al: Osteopontin identified as lead marker of colon cancer progression, using pooled sample expression profiling, J Natl Cancer Inst 94: 513-521, 2002.

23. Novina CD and Sharp PA: The RNAi revolution. Nature 430: 161-164, 2004.

24. Dave RS and Pomerantz RJ: RNA interference: on the road to an alternate therapeutic strategy. Rev Med Virol 13: 373-385, 2003.

25. Sternlicht MD and Werb Z: How matrix metalloproteinases regulate cell behavior. Annu Rev Cell Dev Biol 17: 463-516, 2001.

26. Berge G, Pettersen S, Grotterød I, Bettum IJ, Boye K and Mælandsmo GM: Osteopontin-an important downstream effector of S100A4-mediated invasion and metastasis. In J Cancer 129: 780-790, 2011. 